\title{
Communication system improvement for air traffic service to provide a continuous surveillance over the oceanic area
}

\begin{abstract}
Air traffic service is an extra-ventricular service which regulates and assists aircraft in real time to ensure their safe operations. The importance of the air traffic service is to prevent collision between aircraft and to expedite and maintain an orderly flow of the air traffic. The objective of this project is to assess the effectiveness of the installed system in assisting the air traffic controller in providing the air traffic service. In order to achieve this objective, the Automatic Dependent Surveillance and Controller Pilot Data Link Communication (ADS/CPDLC) system has been installed and its functionality to provide continuous surveillance and communication has been evaluated. A survey of 35 air traffic controllers had participated to assess the effectiveness of the system to the air traffic controller. The results showed that over $80 \%$ of the air traffic controllers agree that the system is able to provide a continuous surveillance and continuous communication over the oceanic area. Apart from that, $60 \%$ of the air traffic controllers agree the reduced separation minima can be applies over the oceanic area by using the application of the system. Base on this result, reduced separation minima over the oceanic area can be applied in order to expedite and maintain an orderly flow of air traffic over the oceanic area.
\end{abstract}

Keyword: Air traffic control; Automatic dependent surveillance; Communication system 